\title{
BUILDING PERFORMANCE SIMULATION MODELING TECHNIQUES
}

\author{
${ }^{1}$ Gábor KÖVÁRI, ${ }^{2}$ István KISTELEGDI Jr \\ Department of Energydesign, Faculty of Engineering and Information Technology, \\ University of Pécs, Boszorkány u. 2, H-7624 Pécs, Hungary \\ e-mail: ${ }^{1}$ koevari.gabor@gmail.com, ${ }^{2}$ kistelegdisoma@ mik.pte.hu
}

Received 14 September 2015; accepted 8 February 2016

\begin{abstract}
This research aims at demonstrating techniques in complex dynamic building energy simulation methods that greatly reduce the otherwise very time-consuming - in particular cases even week-long - computation time of simulation models, however marginal difference arises in the energy results of the simulations. Different test simulations were created to examine how to simplify the models without altering the energy and comfort results, which lead to reduce also the working hours spent on building the model. The building physics behavior of the zones, heating and cooling equipment in the complex model were studied and tested to understand how those can be used, merged or simplified in order to speed up both computation time and model building phase. The IDA ICE complex dynamic building energy simulation program offers two methods - periodic and dynamic - for calculation, which were compared in this program. Test simulations provide information about possible differences between the results of these calculation methods, in order to define the appropriate use of these methods.
\end{abstract}

Keywords: Dynamic building energy simulation, Building physics modeling techniques, Energy-efficiency, Energy optimization, Energy management, Building services systems

\section{Investigation goals, test simulation set up}

Today's most accurate and developed building design supporting tools are dynamic building physics simulations with significantly better and complex modeling of real physical systems than steady state calculations [1]. There are already ongoing research studies in Hungary about the energy performance and energy optimization potential of buildings, which use this different dynamic thermal building simulation methods, coupled with building monitoring measurements [2], [3], [4], [5]. Beside general 
overviews of building thermal energy simulation techniques [6], [7] also building energy performance validation supporting simulation experiences possess first evidence for advanced sustainable building simulations [8], [9]. However, there are few publications about applied experiences about dynamic and periodic calculation time demand. Therefor the subject of the research is to find answers to the question on how can calculation and model building time be reduced, whilst keeping the difference of the energy results of the simulation as low as possible. Test results proved that a welladjusted model is able to eventuate less than $0.02 \%$ difference in the calculation outcome, while its computation time can be reduced even up to $1 / 100$.

Table I shows the simplified steps of the building energy and comfort simulation. Even though the preparation of the energy model (step 2) is just one single step along this roadmap (Table I), the process will determine not only the computation time - that can be even week-long - but also the outcome of the whole simulation. During the construction of the model each and every parameter must be set up very consciously, in order to avoid any unnecessary calculation iteration, time consuming over-detailing, however over-simplification of the model can cause undesirable difference in the final results. Calculation methods (periodic or dynamic) must be chosen in accordance to the purpose of the simulation, respectively investigation.

A periodic simulation means a certain period is simulated a number of times until the system has stabilized and no longer changes from simulation to simulation, a dynamic simulation means that the simulation starts at a particular time and ends at another time. A startup phase may precede the main (calculation) phase. A startup-phase is a simulation with the sole purpose of selecting a suitable starting point for the real experiment. The startup phase also preheats the thermal mass of the building structures in the simulation model.

\section{Table I}

Simplified algorithmic steps of the building energy and comfort simulation

\begin{tabular}{|l|}
\hline $\begin{array}{l}\text { 1. Collecting and systemizing all available plans, details and data of the building to be } \\
\text { simulated; }\end{array}$ \\
\hline 2. Creating the energy model in the IDA ICE software [10]; \\
\hline 3. Setting up the parameters for building energy and climate simulation; \\
\hline 4. Running one-year simulations; \\
\hline 5. Results can be compared with the measured data; \\
\hline 6. Recalibrate the model; \\
\hline 7. Test the concepts for the improvement; \\
\hline 8. Model is validated to work effectively, apply to real building control. \\
\hline
\end{tabular}

\subsection{Test building simulation defaults}

To set up the parameters of the test building correctly the purpose of the simulation must be determined first. This is to find the fastest and most accurate simulation method. While searching this technique there are several questions to be answered: 
1. Can similar zones merged together?

2. Can similar heating equipment merged together?

3. Are there any similarities in merging water radiators, floor-heating or fan-coils?

4. Whether dynamic or periodic simulations provide the most punctual results?

5. While using the periodic calculation method the maximum periodicity must be used or fewer repetitions could provide similarly correct outcome?

6. Are there any laws between the reps and the accuracy of the final result?

7. What is the best method to get quick and accurate result for comfort studies?

Unfortunately, no single method exists to answer all these questions at once. If this simple statement has been accepted, it leads to the recognition that several tests must be run, which means that the test building itself needs to be as simple as possible, yet all the parameters tested should be included and being able to changed easily.

The test building is a resembling of a simplified average family house: $10.0 \times 15.0 \mathrm{~m}, 150.0 \mathrm{~m}^{2}$ floor area, rectangular shape, $2.6 \mathrm{~m}$ clear height, one-story house, with the longer facades facing south and north. 4 pieces of $125 \times 150 \mathrm{~cm}$ widows has been placed on the south façade, while one facing east and west to grant different solar gain during the 1-year period.

To simulate the behavior of the zone-merging two types of a floor plan have been created. The base floor plan consists one $150 \mathrm{~m}^{2}$ zone, while the other has 4 pieces of $37.5 \mathrm{~m}^{2}$ (total of $150 \mathrm{~m}^{2}$ ) (Fig. 1).

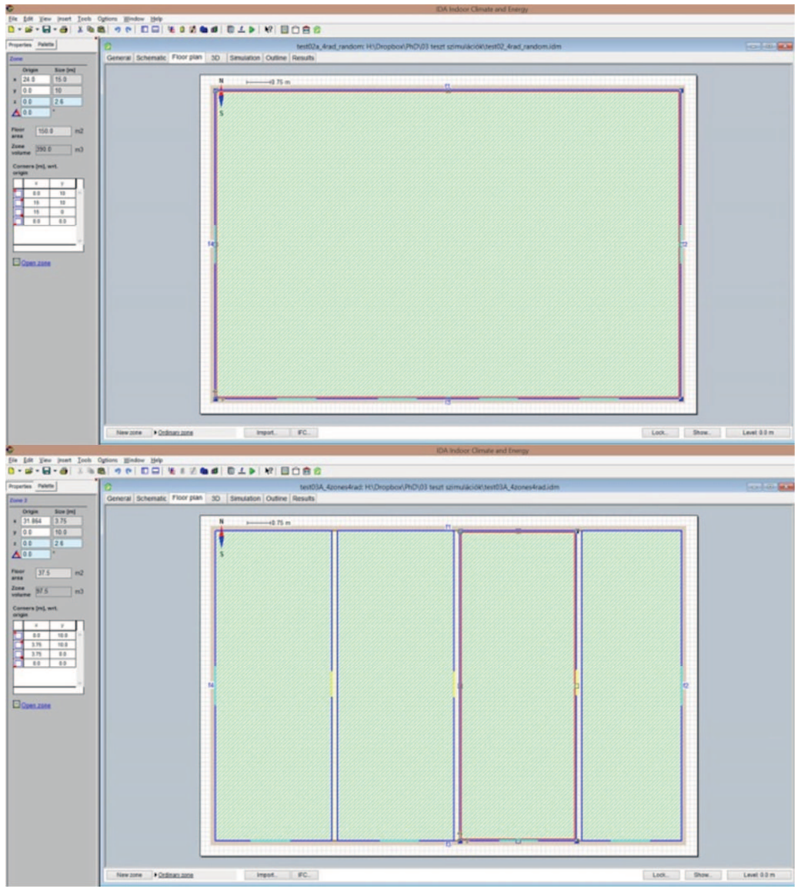

Fig. 1. Floor plans of the test building 
The applied structures and measures of the outer and inner walls, windows, openings and heating equipment are the most commonly used ones. In the 4 zones layout one $50 \mathrm{~W}$ lighting and one $150 \mathrm{~W}$ equipment has been placed in each zone, while in the single zone layout a unit of 4 pieces of light $(4 \times 12.5 \mathrm{~W})$ and equipment $(4 \times 37.5 \mathrm{~W})$ provide the same consumption and thermal load (Table II).

\section{Table II}

Test building simulation defaults

\begin{tabular}{|c|}
\hline 1. Net floor area $10.0 \mathrm{~m} \mathrm{x} 15.0 \mathrm{~m}, 150 \mathrm{~m}^{2}$; \\
\hline 2. Net Volume: $390 \mathrm{~m}^{3}$; \\
\hline 3. Outer walls: $30 \mathrm{~cm}$ brick $+10 \mathrm{~cm}$ EPS; \\
\hline 4. Inner walls: $10 \mathrm{~cm}$ gypsum, openings $100 / 210$ \\
\hline 5. 6 pieces windows: $125 / 150$, double glazing, LOW-E; \\
\hline $\begin{array}{l}\text { 6.1. Water radiators: } 1 \text { piece } 4000 / 900 \mathrm{~mm} 4000 \mathrm{~W} \text {, or } 4 \text { pieces } 1000 / 900 \\
1000 \mathrm{~W} \text {; }\end{array}$ \\
\hline 6.2. Floor heating $150 \mathrm{~m}^{2}$, or 4 pieces of $37.5 \mathrm{~m}^{2}$ \\
\hline $\begin{array}{l}\text { 6.3. Fan-coil (heating) total heat transfer } 12000 \mathrm{~W} \text {, fan-coil (cooling) } \\
\text { total/sensible heat transfer } 12000 \mathrm{~W} \text { or } 4 \text { pieces fan-coil (heating) total heat } \\
\text { transfer each } 3000 \mathrm{~W}, 4 \text { pieces fan-coil (cooling) total/sensible heat } \\
\text { transfer each } 3000 \mathrm{~W} \text {; }\end{array}$ \\
\hline 7. Default cooler: electric chiller COP 3 (theoretical 'unlimited' capacity); \\
\hline 8. Default heater: fuel boiler COP 0.9 (theoretical 'unlimited' capacity); \\
\hline $\begin{array}{l}\text { 9. Default AHU: Standard AHU (theoretical 'unlimited' capacity) with } \\
\text { heat recovery }(\mathrm{eta}=0.6) \text {; }\end{array}$ \\
\hline 10. 4 light sources $50 \mathrm{~W}$ each, always on; \\
\hline 11.4 equipment $150 \mathrm{~W}$ each, always on; \\
\hline 12. Location: Budapest; \\
\hline 13. Weather data: Debrecen (ASHRAE IWEC 1.1) [11]. \\
\hline
\end{tabular}

\subsection{Simulation settings - water radiator}

To test the behavior of a water radiator heat system 6 test simulations were introduced. In each test only a single parameter has been changed in order to keep track of changes in the results. In test01 the whole layout consist of one zone in where 1 piece of a $4.0 \mathrm{~m}$ long, $0.9 \mathrm{~m}$ high, $4000 \mathrm{~W}$ heat output water radiator were placed randomly in the zone.

In test 02 the $150 \mathrm{~m}^{2}$ zone has not been modified, but 4 pieces of $1.0 \mathrm{~m}$ long, $0.9 \mathrm{~m}$ high and $1000 \mathrm{~W}$ heat output radiators were used. In test03 4 zones, (each $\left.150 \mathrm{~m}^{2} / 4=37.5 \mathrm{~m}^{2}\right)$ and 4 pieces of radiators $(1.0 \mathrm{~m} \times 0.9 \mathrm{~m}, 1000 \mathrm{~W})$ in each zone were applied. In IDA ICE radiators can be placed on the exact spot on the wall, such as in real life. To examine its effect on the outcome, we introduced test $01 \mathrm{a}$, test $02 \mathrm{a}$ and test 03 a accordingly to original tests. 


\subsection{Simulation settings - floor heating}

For testing the floor heating system the same test building's layout have been used like in test01 to test03 (Fig. 2). In test04 a $150 \mathrm{~m}^{2}$ size, $26.667 \mathrm{~W} / \mathrm{m}^{2}$ floor heating were placed, while in test04 and test05 4 pieces of a $37.5 \mathrm{~m}^{2} 26.667 \mathrm{~W} / \mathrm{m}^{2}$ floor heating were operated in a single zone (test05) or separately in 4 zones (test06). Total heating power of the system have been calculated $\left(150 \mathrm{~m}^{2} \times 26.667 \mathrm{~W} / \mathrm{m}^{2}=4000 \mathrm{~W}\right)$ to be equal to the water radiator heating in order to gain information on compering those systems (Fig. 3).

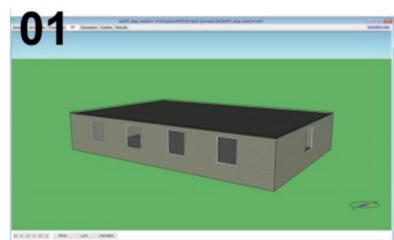

1 zone, 1 radiator, random
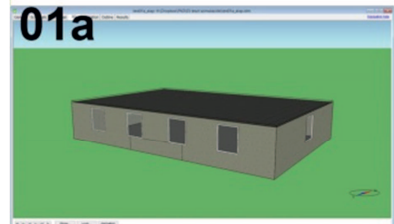

1 zone, 1 radiator, on the wall

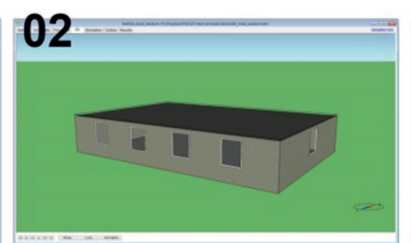

1 zone, 4 radiators, random

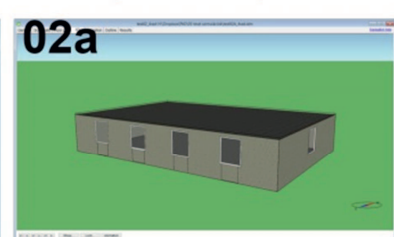

1 zone, 4 radiators, on the wal

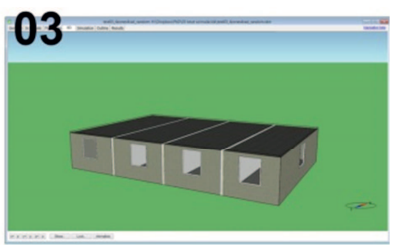

4 zones, 4 radiators, random

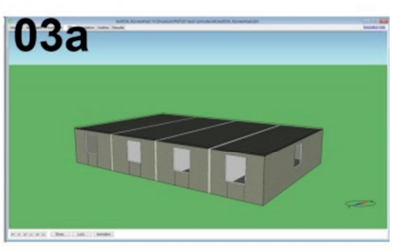

4 zones, 4 radiators, on the wall

Fig. 2. Test simulation scenarios for water radiator heating

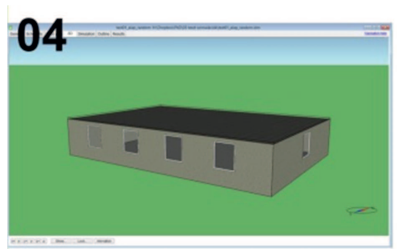

1 zone, 1 floorheating

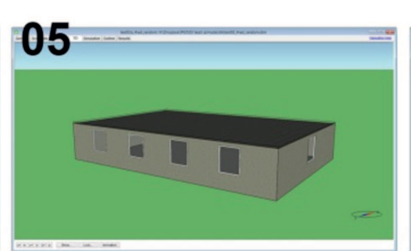

1 zone, 4 floorheating

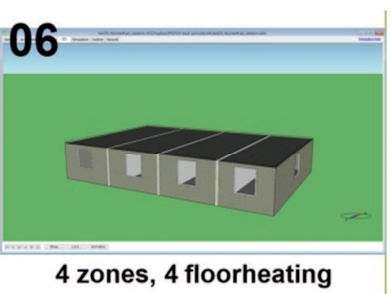

4 zones, 4 floorheating

Fig. 3. Test simulation scenarios for floor heating

\subsection{Simulation settings - fan-coil heating and cooling}

When testing the fan-coil heating and cooling system (test07-test09) (Fig. 4) simulations proved that using a $4000 \mathrm{~W}$ heating and cooling power equipment will result a very high number of dissatisfied (IV. unacceptable comfort category) when examining the EN 15251 [12] thermal comfort. In order that the results of comfort and energy consumption will be similar to the water radiator and floor heating systems $12000 \mathrm{~W}$ power fan-coil was used. Heating fan coil was operated form $1^{\text {st }}$ of October till $31^{\text {st }}$ March, while cooling fan-coil was constantly on from $1^{\text {st }}$ April till $30^{\text {th }}$ September. 


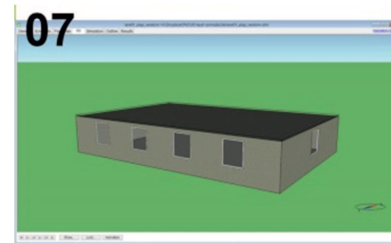

1 zone, 1 Fan-coil

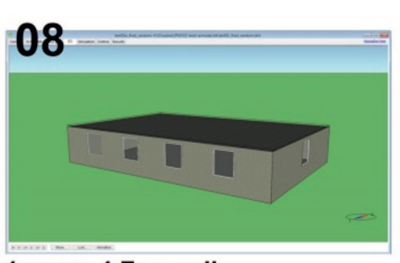

1 zone, 4 Fan-coil

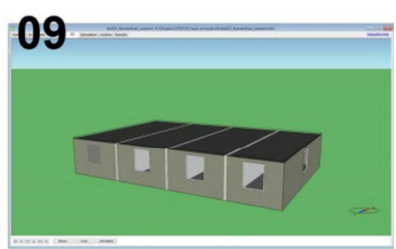

4 zones, 4 Fan-coils

Fig. 4. Test simulation scenarios for fan-coil heating and cooling

\section{Examination of the results of simulation}

Every test simulation scenarios have been run 5 times in both periodic and dynamic method and the average of the computation time and total delivered (final) energy are summarized in Table III, IV, $V$. Maximum value of the periodicity can be chosen freely when running periodic simulation. In order to be able to examine the difference between the results of different periodicity, maximum periodicity has been set to 1, 5, 10,50 and 100 . The actual maximum periodicity has been reached is recorded.

Table VI, VII, VIII demonstrate the different heating systems in the models with regard to indoor comfort qualities (comfort categories according to EN 15251) [4] in dependence of the variable heat transfer methods.

Table III

Simulation results -Final energy

\begin{tabular}{|c|c|c|c|c|c|c|c|c|c|c|}
\hline & \multicolumn{9}{|c|}{ Dynamic vs periodic } \\
\hline & & \multicolumn{3}{|c|}{ Dynamic } & \multicolumn{6}{|c|}{ Periodic } \\
\hline & & & 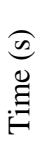 & 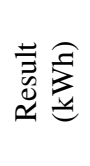 & & 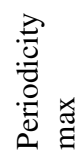 & 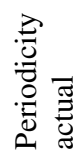 & 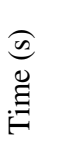 & 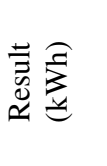 & 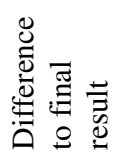 \\
\hline \multirow{15}{*}{ 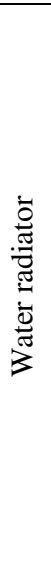 } & \multirow{5}{*}{ 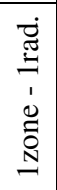 } & \multirow{5}{*}{ 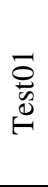 } & \multirow{5}{*}{26} & \multirow{5}{*}{28150} & \multirow{5}{*}{ 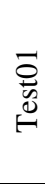 } & 1 & 1 & 25 & 28320 & $0.11 \%$ \\
\hline & & & & & & 5 & 5 & 128 & 28288 & $0.00 \%$ \\
\hline & & & & & & 10 & 6 & 153 & 28288 & \\
\hline & & & & & & 50 & - & - & - & \\
\hline & & & & & & 100 & - & - & - & \\
\hline & \multirow{5}{*}{ 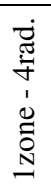 } & \multirow{5}{*}{ 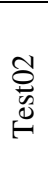 } & \multirow{5}{*}{28} & \multirow{5}{*}{28162} & \multirow{5}{*}{ 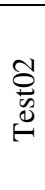 } & 1 & 1 & 28 & 28322 & $0.12 \%$ \\
\hline & & & & & & 5 & 5 & 137 & 28289 & $0.00 \%$ \\
\hline & & & & & & 10 & 5 & 137 & 28289 & \\
\hline & & & & & & 50 & - & - & - & \\
\hline & & & & & & 100 & - & - & - & \\
\hline & \multirow{5}{*}{ 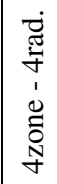 } & \multirow{5}{*}{ 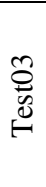 } & \multirow{5}{*}{51} & \multirow{5}{*}{28183} & \multirow{5}{*}{ 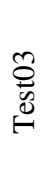 } & 1 & 1 & 50 & 28315 & $0.03 \%$ \\
\hline & & & & & & 5 & 5 & 247 & 28310 & $0.01 \%$ \\
\hline & & & & & & 10 & 8 & 391 & 28307 & \\
\hline & & & & & & 50 & - & - & - & \\
\hline & & & & & & 100 & - & - & - & \\
\hline
\end{tabular}

Pollack Periodica 11, 2016, 2 
Table IV

Simulation results - Final energy

\begin{tabular}{|c|c|c|c|c|c|c|c|c|c|c|}
\hline & \multicolumn{9}{|c|}{ Dynamic vs periodic } \\
\hline & & \multicolumn{3}{|c|}{ Dynamic } & \multicolumn{6}{|c|}{ Periodic } \\
\hline & & & $\begin{array}{l}\text { Time } \\
\text { (s) }\end{array}$ & $\begin{array}{l}\text { Result } \\
(\mathrm{kWh})\end{array}$ & & $\begin{array}{l}\text { Periodicity } \\
\max \end{array}$ & $\begin{array}{l}\text { Periodicity } \\
\text { actual }\end{array}$ & $\begin{array}{c}\text { Time } \\
\text { (s) }\end{array}$ & $\begin{array}{l}\text { Result } \\
(\mathrm{kWh})\end{array}$ & $\begin{array}{l}\text { Difference } \\
\text { to final } \\
\text { result }\end{array}$ \\
\hline \multirow{15}{*}{ 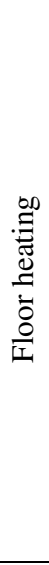 } & \multirow{5}{*}{\multicolumn{2}{|c|}{ 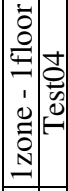 }} & \multirow{5}{*}{32} & \multirow{5}{*}{29320} & \multirow{5}{*}{ 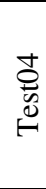 } & 1 & 1 & 32 & 29523 & $0.52 \%$ \\
\hline & & & & & & 5 & 5 & 156 & 29374 & $0.01 \%$ \\
\hline & & & & & & 10 & 10 & 310 & 29372 & $0.01 \%$ \\
\hline & & & & & & 50 & 18 & 679 & 29370 & \\
\hline & & & & & & 100 & - & - & - & \\
\hline & \multirow{5}{*}{\multicolumn{2}{|c|}{ 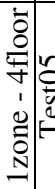 }} & \multirow{5}{*}{38} & \multirow{5}{*}{29421} & \multirow{5}{*}{ 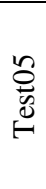 } & 1 & 1 & 38 & 29497 & $0.45 \%$ \\
\hline & & & & & & 5 & 5 & 189 & 29361 & $0.01 \%$ \\
\hline & & & & & & 10 & 10 & 375 & 29363 & $0.01 \%$ \\
\hline & & & & & & 50 & 16 & 697 & 29365 & \\
\hline & & & & & & 100 & - & - & - & \\
\hline & \multirow{5}{*}{\multicolumn{2}{|c|}{ 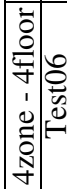 }} & \multirow{5}{*}{64} & \multirow{5}{*}{29153} & \multirow{5}{*}{ 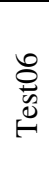 } & 1 & 1 & 63 & 29322 & $0.38 \%$ \\
\hline & & & & & & 5 & 5 & 310 & 29207 & $0.01 \%$ \\
\hline & & & & & & 10 & 10 & 622 & 29209 & $0.01 \%$ \\
\hline & & & & & & 50 & 21 & 1307 & 29211 & \\
\hline & & & & & & 100 & - & - & - & \\
\hline
\end{tabular}

Table V

Simulation results - Final energy

\begin{tabular}{|c|c|c|c|c|c|c|c|c|c|c|}
\hline & \multicolumn{9}{|c|}{ Dynamic vs periodic } \\
\hline & & \multicolumn{3}{|c|}{ Dynamic } & \multicolumn{6}{|c|}{ Periodic } \\
\hline & & & $\begin{array}{l}\text { Time } \\
\text { (s) }\end{array}$ & $\begin{array}{l}\text { Result } \\
(\mathrm{kWh})\end{array}$ & & $\begin{array}{l}\text { Periodicity } \\
\max \end{array}$ & $\begin{array}{l}\text { Periodicity } \\
\text { actual }\end{array}$ & $\begin{array}{l}\text { Time } \\
\text { (s) }\end{array}$ & $\begin{array}{l}\text { Result } \\
(\mathrm{kWh})\end{array}$ & $\begin{array}{l}\text { Difference } \\
\text { to final } \\
\text { result }\end{array}$ \\
\hline \multirow{15}{*}{ 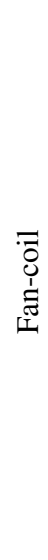 } & \multirow{5}{*}{ 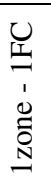 } & \multirow{5}{*}{ 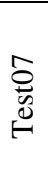 } & \multirow{5}{*}{75} & \multirow{5}{*}{27708} & \multirow{5}{*}{$\hat{0}$} & 1 & 1 & 69 & 27275 & $0.16 \%$ \\
\hline & & & & & & 5 & 5 & 345 & 27230 & $0.01 \%$ \\
\hline & & & & & & 10 & 10 & 669 & 27231 & $0.00 \%$ \\
\hline & & & & & & 50 & 26 & 1699 & 27232 & \\
\hline & & & & & & 100 & - & - & - & \\
\hline & \multirow{5}{*}{ 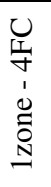 } & \multirow{5}{*}{ 离 } & \multirow{5}{*}{75} & \multirow{5}{*}{27734} & \multirow{5}{*}{ 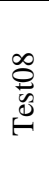 } & 1 & 1 & 78 & 27902 & $0.03 \%$ \\
\hline & & & & & & 5 & 5 & 380 & 27892 & $0.01 \%$ \\
\hline & & & & & & 10 & 10 & 753 & 27895 & $0.01 \%$ \\
\hline & & & & & & 50 & 26 & 1355 & 27895 & \\
\hline & & & & & & 100 & - & - & - & \\
\hline & \multirow{5}{*}{ 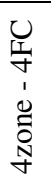 } & \multirow{5}{*}{ 嶽 } & \multirow{5}{*}{392} & \multirow{5}{*}{27369} & \multirow{5}{*}{ 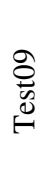 } & 1 & 1 & 413 & 27541 & $0.01 \%$ \\
\hline & & & & & & 5 & 5 & 1984 & 27542 & $0.01 \%$ \\
\hline & & & & & & 10 & 10 & 3914 & 27545 & $0.00 \%$ \\
\hline & & & & & & 50 & 22 & 8404 & 27544 & \\
\hline & & & & & & 100 & - & - & - & \\
\hline
\end{tabular}


Table VI

Simulation results - Comfort

\begin{tabular}{|c|c|c|c|c|c|c|c|c|c|}
\hline & \multicolumn{8}{|c|}{ Dynamic vs periodic with comfort } \\
\hline & & \multicolumn{4}{|c|}{ Dynamic } & \multicolumn{4}{|c|}{ Periodic } \\
\hline & & & $\begin{array}{c}\text { Time } \\
\text { (s) }\end{array}$ & $\begin{array}{l}\text { PPD } \\
\text { PMV } \\
(\text { mean*8760h) }\end{array}$ & $\begin{array}{l}\text { Comfort } \\
\text { category } \\
\text { (EN15251) }\end{array}$ & & $\begin{array}{l}\text { Time } \\
\text { (s) }\end{array}$ & $\begin{array}{l}\text { PPD } \\
\text { PMV } \\
(\text { mean*8760h) }\end{array}$ & $\begin{array}{l}\text { Comfort } \\
\text { category } \\
\text { (EN15251) }\end{array}$ \\
\hline \multirow{12}{*}{ 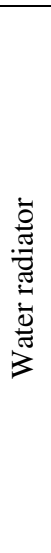 } & \multirow{4}{*}{ 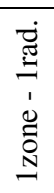 } & \multirow{4}{*}{ 家 } & \multirow{4}{*}{27} & \multirow{4}{*}{$\begin{array}{c}106283.6 \\
-4939.3\end{array}$} & 1938 (I.) & \multirow{4}{*}{ 勇 } & \multirow{4}{*}{167} & \multirow{4}{*}{$\begin{array}{c}106739.5 \\
-4954.2\end{array}$} & 1854 (I.) \\
\hline & & & & & 5525 (II.) & & & & 5525 (II.) \\
\hline & & & & & 6304 (III.) & & & & 6299 (III.) \\
\hline & & & & & 2456 (IV.) & & & & 2461 (IV.) \\
\hline & \multirow{4}{*}{ 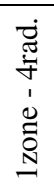 } & \multirow{4}{*}{ 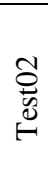 } & \multirow{4}{*}{28} & \multirow{4}{*}{$\begin{array}{c}106279.4 \\
-4938.6\end{array}$} & 1936 (I.) & \multirow{4}{*}{ 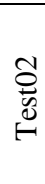 } & \multirow{4}{*}{138} & \multirow{4}{*}{$\begin{array}{c}106756.1 \\
-4954.7\end{array}$} & 1874 (I.) \\
\hline & & & & & 5525 (II.) & & & & 5549 (II.) \\
\hline & & & & & 6299 (III.) & & & & 6321 (III.) \\
\hline & & & & & 2461 (IV.) & & & & 2439 (IV.) \\
\hline & \multirow{4}{*}{ 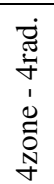 } & \multirow{4}{*}{ 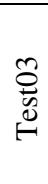 } & \multirow{4}{*}{52} & \multirow{4}{*}{$\begin{array}{c}105928.9 \\
-4934.6\end{array}$} & 3469 (I.) & \multirow{4}{*}{ ڤొ } & \multirow{4}{*}{399} & \multirow{4}{*}{$\begin{array}{c}106431.9 \\
-4869.2\end{array}$} & 3256 (I.) \\
\hline & & & & & 5520 (II.) & & & & 5544 (II.) \\
\hline & & & & & 5929 (III.) & & & & 5953 (III.) \\
\hline & & & & & 2831 (IV.) & & & & 2807 (IV.) \\
\hline
\end{tabular}

Table VII

Simulation results - Comfort

\begin{tabular}{|c|c|c|c|c|c|c|c|c|c|}
\hline & \multicolumn{8}{|c|}{ Dynamic vs periodic with comfort } \\
\hline & & \multicolumn{4}{|c|}{ Dynamic } & \multicolumn{4}{|c|}{\begin{tabular}{|l|} 
Periodic \\
\end{tabular}} \\
\hline & & & $\begin{array}{l}\text { Time } \\
(\mathrm{s})\end{array}$ & $\begin{array}{l}\text { PPD } \\
\text { PMV } \\
(\text { mean*8760h) }\end{array}$ & \begin{tabular}{|l|} 
Comfort \\
category \\
(EN15251) \\
\end{tabular} & & $\begin{array}{l}\text { Time } \\
(\mathrm{s})\end{array}$ & $\begin{array}{l}\text { PPD } \\
\text { PMV } \\
(\text { mean*8760h) }\end{array}$ & $\begin{array}{l}\text { Comfort } \\
\text { category } \\
\text { (EN15251) }\end{array}$ \\
\hline \multirow{12}{*}{$\frac{\stackrel{0}{0}}{\stackrel{\Xi}{\Xi}}$} & \multirow{4}{*}{ 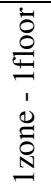 } & \multirow{4}{*}{ 志 } & \multirow{4}{*}{32} & \multirow{4}{*}{$\begin{array}{l}94337.7 \\
-4487.8\end{array}$} & 5544 (I.) & \multirow{4}{*}{ 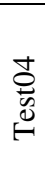 } & \multirow{4}{*}{802} & \multirow{4}{*}{$\begin{array}{l}94337.9 \\
-4487.6\end{array}$} & 5544 (I.) \\
\hline & & & & & 5552 (II.) & & & & 5552 (II.) \\
\hline & & & & & 6329 (III.) & & & & 6329 (III.) \\
\hline & & & & & 2431 (IV.) & & & & 2431 (IV.) \\
\hline & \multirow{4}{*}{ 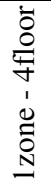 } & \multirow{4}{*}{ 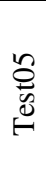 } & \multirow{4}{*}{38} & \multirow{4}{*}{$\begin{array}{l}94341.7 \\
-4488.2\end{array}$} & 5544 (I.) & \multirow{4}{*}{ 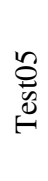 } & \multirow{4}{*}{697} & \multirow{4}{*}{$\begin{array}{l}94344.6 \\
-4488.4\end{array}$} & 5544 (I.) \\
\hline & & & & & 5552 (II.) & & & & 5552 (II.) \\
\hline & & & & & 6327 (III.) & & & & 6328 (III.) \\
\hline & & & & & 2433 (IV.) & & & & 2432 (IV.) \\
\hline & \multirow{4}{*}{ 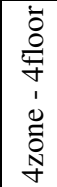 } & \multirow{4}{*}{ 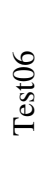 } & \multirow{4}{*}{64} & \multirow{4}{*}{$\begin{array}{l}96315.9 \\
-4431.4\end{array}$} & 5526 (I.) & \multirow{4}{*}{ 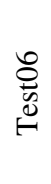 } & \multirow{4}{*}{1315} & \multirow{4}{*}{$\begin{array}{l}96318.9 \\
-4564.1\end{array}$} & 5547 (I.) \\
\hline & & & & & 6366 (II.) & & & & 6369 (II.) \\
\hline & & & & & 8320 (III.) & & & & 8319 (III.) \\
\hline & & & & & 440 (IV.) & & & & 441 (IV.) \\
\hline
\end{tabular}

Pollack Periodica 11, 2016, 2 
Table VIII

Simulation results - Comfort

\begin{tabular}{|c|c|c|c|c|c|c|c|c|c|}
\hline & \multicolumn{8}{|c|}{ Dynamic vs periodic with comfort } \\
\hline & & \multicolumn{4}{|c|}{ Dynamic } & \multicolumn{4}{|c|}{$\begin{array}{l}\text { Periodic } \\
\end{array}$} \\
\hline & & & $\begin{array}{l}\text { Time } \\
(\mathrm{s})\end{array}$ & $\begin{array}{l}\text { PPD } \\
\text { PMV } \\
(\text { mean*8760h) }\end{array}$ & $\begin{array}{l}\text { Comfort } \\
\text { category } \\
\text { (EN15251) }\end{array}$ & & $\begin{array}{l}\text { Time } \\
\text { (s) }\end{array}$ & $\begin{array}{l}\text { PPD } \\
\text { PMV } \\
(\text { mean*8760h) }\end{array}$ & $\begin{array}{l}\text { Comfort } \\
\text { category } \\
\text { (EN15251) }\end{array}$ \\
\hline \multirow{13}{*}{$\begin{array}{l}\overline{\bar{\delta}} \\
0 \\
i \\
\stackrel{\Xi}{ \pm} \\
\text { II }\end{array}$} & \multirow{4}{*}{ 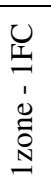 } & \multirow{4}{*}{ 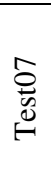 } & \multirow{4}{*}{75} & \multirow{4}{*}{$\begin{array}{c}122077.2 \\
-5492.9\end{array}$} & 304 (I.) & \multirow{4}{*}{ 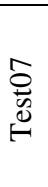 } & \multirow{4}{*}{1699} & \multirow{4}{*}{$\begin{array}{c}122232.2 \\
-5496.6\end{array}$} & 289 (I.) \\
\hline & & & & & 4611 (II.) & & & & 4635 (II.) \\
\hline & & & & & 6183 (III.) & & & & 6209 (III.) \\
\hline & & & & & 2577 (IV.) & & & & 2551 (IV.) \\
\hline & \multirow{4}{*}{ 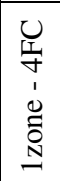 } & \multirow{4}{*}{ 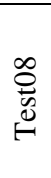 } & \multirow{4}{*}{75} & \multirow{4}{*}{$\begin{array}{c}120498.5 \\
-5442.0\end{array}$} & 383 (I.) & \multirow{4}{*}{\begin{tabular}{l}
$\infty$ \\
0 \\
\multirow{0}{0}{} \\
$\stackrel{0}{\oplus}$
\end{tabular}} & \multirow{4}{*}{1355} & \multirow{4}{*}{$\begin{array}{c}120626.3 \\
-5448.1\end{array}$} & 366 (I.) \\
\hline & & & & & 4626 (II.) & & & & 4613 (II.) \\
\hline & & & & & 6206 (III.) & & & & 6184 (III.) \\
\hline & & & & & 2554 (IV.) & & & & 2576 (IV.) \\
\hline & \multirow{5}{*}{ 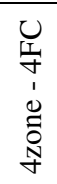 } & \multirow{5}{*}{ 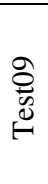 } & \multirow{5}{*}{392} & \multirow{5}{*}{$\begin{array}{c}118255.0 \\
-5518.6\end{array}$} & 487 (I.) & \multirow{5}{*}{ Oे } & \multirow{5}{*}{8404} & \multirow{5}{*}{$\begin{array}{c}123177.3 \\
-5307.0\end{array}$} & 227 (I.) \\
\hline & & & & & 5419 (II.) & & & & 4774 (II.) \\
\hline & & & & & 8544 (III.) & & & & 8122 (III.) \\
\hline & & & & & 216 (IV.) & & & & 638 (IV.) \\
\hline & & & & & & & & & \\
\hline
\end{tabular}

Clear regularities can be seen when the results are shown in graph. Even though in test01 to test03 (periodic calculation method) (Fig. 5) different layout and radiator allocation were used - although total floor area and total heating power were unchanged - difference of the results for total delivered energy was never higher than $0.41 \%$. (28 $288 \mathrm{kWh}-28406 \mathrm{kWh}$ ). However, in terms of processing time, a more than 7.5 times difference can be seen. Notwithstanding, the simplest simulation test01 (one zone, one radiator) was expected to be calculated quickest, the simulations proved different to test02 (one zone, 4 radiators) needed the least amount of computation time.
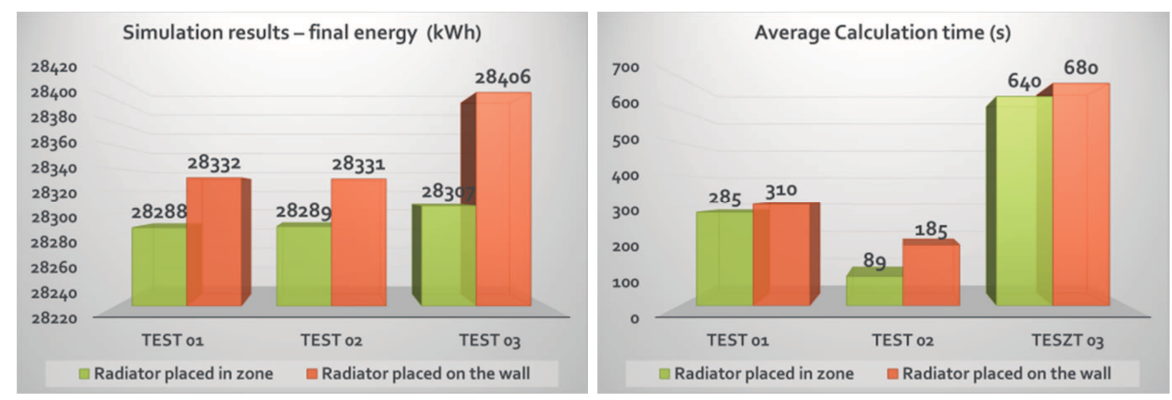

Fig. 5. Final simulation results and calculation time

As different heating system simulations (Fig. 6, Fig. 7 and Fig. 8) are examined the same correlation can be found: final energy results not differ significantly, while the 
same pattern can be seen at computational time as seen with the water radiator system: tests including 1 zone, 4 heat delivery equipment could be calculated faster or at least as fast as test includes 1 zone and 1 heat delivery equipment (Table $I V$ - $V$ ). To calculate tests with 4 zones, 4 heat delivery equipment multiple time will be required than single zone tests.

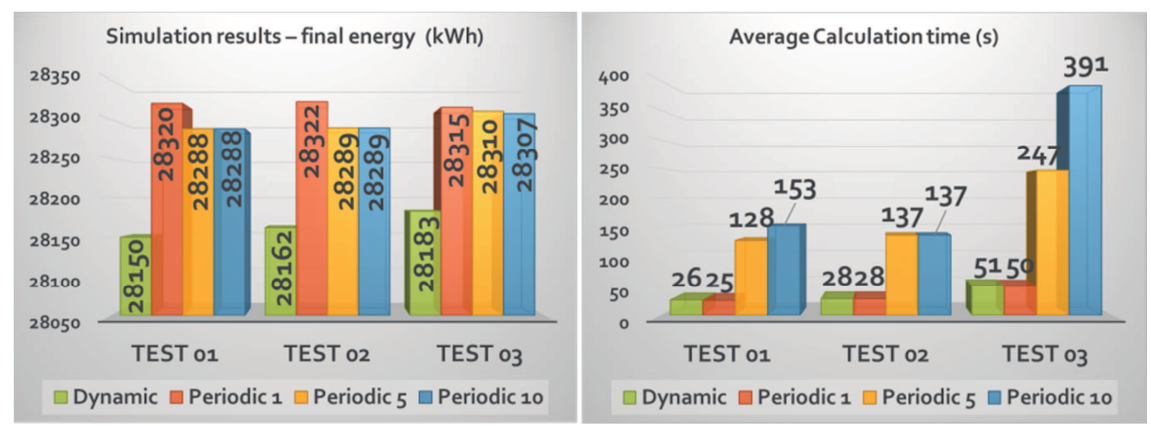

Fig. 6. Simulation results and calculation time - water radiator

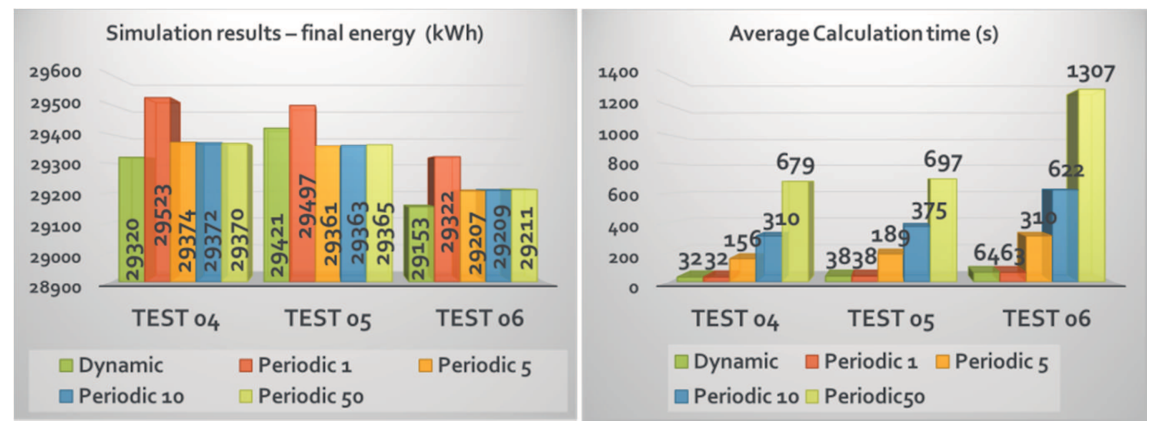

Fig. 7. Simulation results and calculation time - floor heating

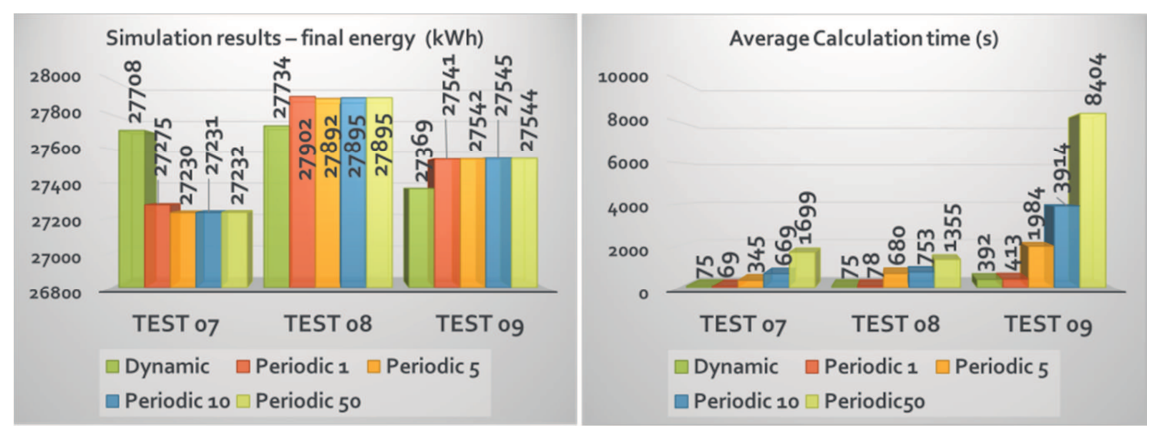

Fig. 8. Simulation results and calculation time - Fan-coil 
IDA ICE complex building simulation software is capable to recalculate results automatically in periodic calculation mode till the preset tolerance level has been reached. The number of repetition can be more than 25 as seen in Table III, IV, $V$. The calculation time is directly proportional to the number of repetitions. The question arises by itself: Whether maximum periodicity must be used in every case or fewer repetition could provide similarly correct outcome? To answer that test04 and test05 were run in dynamic mode and in periodic mode 1 to 5 times and the results are shown in Fig. 9.

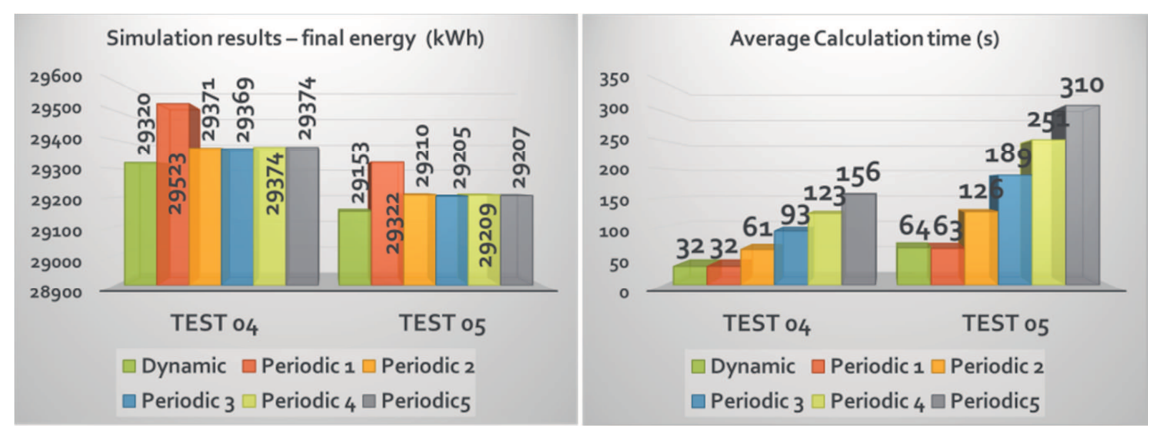

Fig. 9. Effective number of periodicity

It can be clearly stated that calculation time directly proportional to the repetition number and 1 periodic calculation time equal to the dynamic calculation time. It is also apparent that 1 periodic or dynamic calculation will not provide enough accuracy. However, after two repetitions of the calculation the result is nearly constant.

\section{Conclusion}

In order to speed up the model building and computation time, after the analysis of the test simulations carried out the following statements can be made:

- Similar and neighboring zones can be merged together without compromising the calculation result;

- Heat delivery equipment cannot be sum up by using one large equipment, instead of multiple ones. This technique will not decrease calculation time;

- Placing water radiators on the exact spot on the wall will make the model building and calculation longer and more difficult, but will not provide more accurate result;

- For a quick control of the model or for comfort studies dynamic or periodic method with periodicity set to 1 can be used;

- Maximum accuracy can ben reach in periodic method periodicity set to 2. More periodicity will result multiple calculation time but accuracy will not improve;

- The best compromise between quickest calculation and most accurate results delivers periodic simulation with maximum number of periods set to 2 . 


\section{Acknowledgements}

This work is part of the collaboration research project between the Faculty of Engineering and Information Technology, University of Pécs and the Siemens cPlc.

\section{References}

[1] Hensen J. L. M., Lamberts R. (Eds.) Building performance simulation for design and operation, Spon Press, London, 2011.

[2] Kővári K., Kistelegdi I. J. Optimized building automation and control for the improvement of energy efficiency and climate comfort of office buildings, Pollack Periodica, Vol. 10, No. 1, 2015, pp. 71-82.

[3] Baranyai B., Kistelegdi I. Energy management monitoring and control of public buildings, Pollack Periodica, Vol. 9, No. 2, 2014, pp. 77-88.

[4] Póth B., Kistelegdi I. Energy and climate simulations and management system in the Szentágothai research center, Pollack Periodica, Vol. 9, No. 1, 2014, pp. 61-70.

[5] Szodrai F. Simulations of the changes of the heating energy demand and transmission losses of buildings in central European climate, Combination of experiments and simulations, International Review of Applied Sciences and Engineering, Vol. 6, No. 2, 2015, pp. 129-139.

[6] Clarke J. A. Energy simulation in building design, $2^{\text {nd }}$ Edition, University of Strathclyde, Butterworth Heinemann, Glasgow, Scotland, 2001.

[7] Hong T., Chou S. K., Bong T. Y. Building simulation: an overview of developments and information sources, Building and Environment, Vol. 35, No. 4, 2000, pp. 347-361.

[8] Soebarto V. I. Calibration of Hourly energy simulation using Hourly monitored data and monthly utility records for two case study buildings, Department of Architecture Texas A\&M University, Journal Proceedings of Building Simulation, Vol. 9,1997, pp. 411-419, http://www.ibpsa.org/\%5Cproceedings\%5CBS1997\%5CBS97_P017.pdf (last visited 25 August 2015).

[9] Pollock M., Roderick Y., McEwan D., Wheatley C. Building simulation as an assisting tool in designing an energy efficient building, A case study, Eleventh International IBPSA Conference, Glasgow, Scotland, 27-30 July, 2009, http://www.ibpsa.org/proceedings/ bs2009/bs09_1191_1198.pdf, (last visited 25 August 2015).

[10] IDA ICE, indoor climate and energy, EQUA Simulation AB, Solna, Sweden, February 2013, http://www.equaonline.com/iceuser/pdf/ICE45eng.pdf, (last visited 25 August 2015).

[11] ASHRAE, International weather for energy calculation, EQUA Simulation AB, Solna, Sweden, February 2013, ttp://www.equaonline.com/iceuser/ASHRAE_IWEC.html, (last visited 25 August 2015)

[12] EN 15251, Indoor environmental input parameters for design and assessment of energy performance of buildings- addressing indoor air quality, thermal environment, lighting and acoustics, CEN, Brussels, 2007. 